CLAWAR 2019: 22nd International Conference on

Climbing and Walking Robots and the Support

Technologies for Mobile Machines,

Kuala Lumpur, Malaysia, 26-28 August 2019.

https://doi.org/10.13180/clawar.2019.26-28.08.11

\title{
MOTION PLANNING OF A WALKING ROBOT WITH ROTARY MOVERS
}

\author{
E. BRISKIN, A. MALOLETOV, N. SHARONOV \\ Department of Theoretical Mechanics, Volgograd State Technical University, \\ Lenin av., 28, Volgograd, 400005, Russia
}

\begin{abstract}
A method for determining programmed motion laws of a modular multisupport walking machine with several twin movers is considered. Arbitrary planar motion of this machine without slippage of the feet on the ground is studied. The machine control is formed by a matrix of control actions. Simulation results are discussed.
\end{abstract}

\section{Introduction}

There are various types of walking machines that differ from each other in functionality, kinematics of their transmission and movers, control systems, and other parameters $[1,2]$.

Among movers used in walking machines, orthogonal motors are most simple in control and energy efficient $[3,4])$. There disadvantages are that they are relatively large and have prismatic articulations in walking mechanisms. However, the progress in the manufacturing technology of roller-screw and ball-screw linear actuators makes it possible to use these actuators instead of prismatic articulations; the use of kinematics with twin walking mechanisms (as, e.g., in the "Vosminog" machine [5-7]) and common rotary actuator also reduces the dimensions of the mover. An example of the kinematics of a machine with twin orthogonal rotatory (TOR) movers is provided by the "Ortonog" machine [8] shown in Fig. 1.

The TOR mover (Fig. 2) is fixed on the body 1 of the walking machine; it consists of two planar orthogonal walking mechanisms, which have two actuators each-forward (horizontal) motion actuator 3 and adaptation (vertical motion) actuator 2. These actuators are connected by rod 5. Each walking mechanism is equipped with a foot, which is connected with the support pillar by a spherical joint. While the foot of one TOR is in support phase 6 , the other foot is in transfer phase 7. The guides of the forward motion actuators of both mechanisms are connected with each other; due to the common rotary actuator

\footnotetext{
* This work was supported by the RSF (project 18-71-10069).
} 
4, they can rotate relative to the machine's body thus changing the orientation of the walking planes of the orthogonal mechanisms.

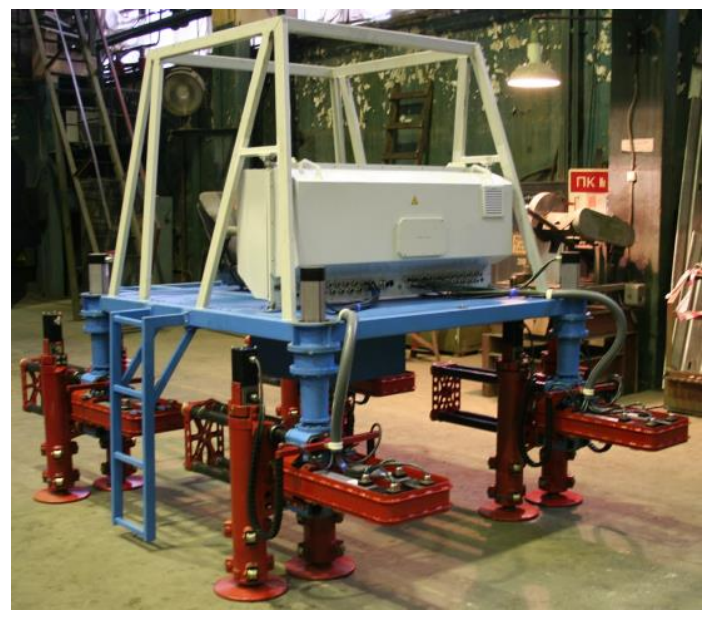

Figure 1. Modular walking machine "Ortonog".

A feature of the twin mover operation is that when one of the walking mechanisms is in transfer phase, the other rests on the ground. If there are a sufficient number of movers, this feature ensures the static stability of the machine.

The use of the common rotary actuator on the one hand gives a sufficient number of controllable degrees of freedom of the machine body and, on the other hand, simplifies the kinematics of the machine because it requires five actuators for two walking mechanisms instead of three gives per each walking mechanism when they are used independently.

Note that an arbitrary movement of any point of the body in the plane of motion under arbitrary orientation of the body is geometrically feasible. The direction of the machine's center velocity and the rotation angle of the body are independent of each other. Traditional vehicles have no such capability even though the use of rotary wheels makes it possible to implement this capability [9] but with slippage when moving on the real-life ground.

The design of the TOR movers makes it possible to decompose the problem of determining the programmed laws of control of the forward motion and adaptation actuators. As a result, the construction and investigation of mathematical models can be simplified by reducing them to the investigation of the planar motion of solid bodies; in this case, the problem of stepping (controlling the adaptation actuators) and moving on the supporting surface 
(controlling the rotation and forward motion actuators) are considered separately.

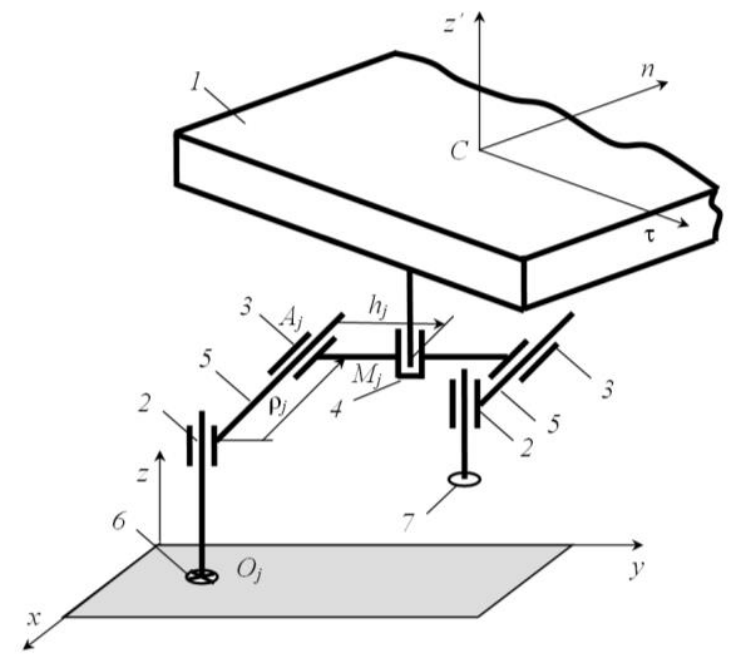

Figure 2. Schematic of the twin orthogonal rotary (TOR) mover

In this paper, we solve the problem of generation of coordinated programmed control laws of the system of actuators of a multisupport walking machine with an arbitrary number of TOR movers that ensure the desired characteristics of the machine motion as it moves on the supporting surface.

\section{CONCLUSIONS}

The kinematics of the walking machine with twin orthogonal rotator movers of the type "Ortonog" makes it possible to execute arbitrary planar motion of the body. For any number of TOR movers and various configurations o the machine, the motion is controlled by specifying three arbitrary components of velocity vector. The proposed method makes it possible to determine the changes in all the other components of velocity vector and equations of programmed motions of the body and actuators.

\section{References}

1. D. E. Okhotsimskii, A. K. Platonov, A. A. Kiril'chenko, et al., "Walking machines," Preprint of the Keldysh Institute of Applied Mathematics, Russian Academy of Sciences (1989). 
2. D. E. Okhotsimskii and Yu. F. Golubev, Mechanics and Control of Motion of an Automatic Walking Machine (Nauka, Moscow, 1984) [in Russian].

3. I. I. Artobolevskii and N. V. Umnov, "Some issues concerning the design of walking machines," Vestn. Akad. Nauk SSSR, No. 2, 22-27 (1969).

4. V. V. Lapshin, Mechanics and Control of Motion of Walking Machines (Bauman Mosc. Gos. Tekhn. Univ., Moscow, 2012) [in Russian]. 